\title{
Feinnadelaspiration peripherer Lymphknoten
}

\author{
Sylvia Gütz, Thomas Knappe
}

\begin{abstract}
Die Feinnadelaspiration ist eine minimalinvasive Technik, um aus einem Lymphknoten Gewebe zur zytologischen Untersuchung zu gewinnen. Die Methode ist schnell einsetzbar, kostengünstig und nahezu schmerzlos für den Patienten. Auch die Risiken sind überschaubar - dennoch sollten Sie tiefer gelegene Lymphknoten unter Ultraschallsicht punktieren.
\end{abstract}

Abb. 1 Verwendete Materialien: Hautdesinfektionsmittel, sterile Tupfer, sterile Handschuhe, Kanülen, 10er Spritze, Objektträger.
Hohe diagnostische Genauigkeit I Die Feinnadelaspiration ermöglicht in kürzester Zeit und mit minimalem technischem Aufwand eine zuverlässige Aussage zu Dignität und Ätiologie peripherer Lymphknotenschwellungen und ist damit eine Weichenstellung für die weiterführende Diagnostik. Mit einer diagnostischen Genauigkeit zwischen 84 und $97 \%$ lassen sich bei richtiger Durchführung maligne Veränderungen von benignen abgrenzen. Die Methode belastet den Patienten kaum.

\section{Bei lymphatischen Systemerkrankungen gestattet die Feinnadelpunktion in aller Regel eine Verdachtsdiagnose. Zur exakten Klassifizierung ist aber eine histologische Beurteilung nötig [1, 3, 4, 5].}

Punktion unter Ultraschallsicht | Oberflächlich gelegene, palpable Lymphknoten können ohne zusätzliche Bildgebung punktiert werden. Das trifft auch für metastasenverdächtige Hautläsionen zu. Die Treffsicherheit bei in der Tiefe, z. B. supraklavikulär oder inguinal lokalisierten Lymph- knoten ist deutlich besser und von weniger Komplikationen begleitet, wenn die Punktion unter Ultraschallsicht durchgeführt wird [1].

\section{Bevor es losgeht}

Indikationen I Eine Feinnadelaspiration ist indiziert bei peripheren Lymphknotenschwellungen, die nicht augenscheinlich durch einen benachbarten Entzündungsprozess hervorgerufen werden. Häufige Ursachen sind:

- maligne lymphatische Systemerkrankungen

- Lymphknotenmetastasen

- solide Tumoren

- Sarkoidose

Material | Verwendet werden feine Nadeln mit einem Durchmesser zwischen 20 und 26 Gauge. Für oberflächlich gelegene Lymphknotenschwellungen eignet sich am besten eine einfache 20er Kanüle mit einer Länge von $20 \mathrm{~mm}$. Bei der ultraschallgestützten Punktion können SpezialanästhesieKanülen mit Mandrin eingesetzt werden. Die weiteren Materialien sind in $\mathbf{A}$ Abb. 1 dargestellt.

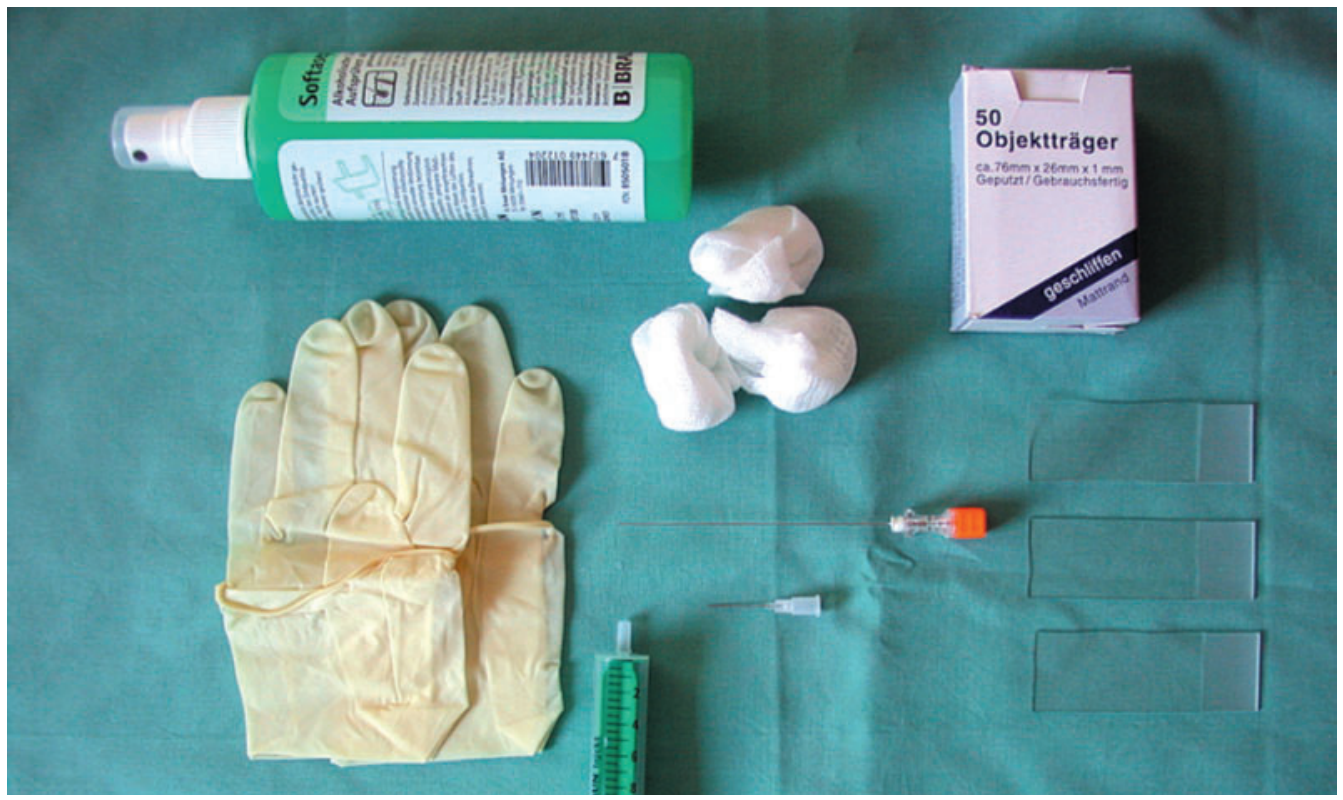



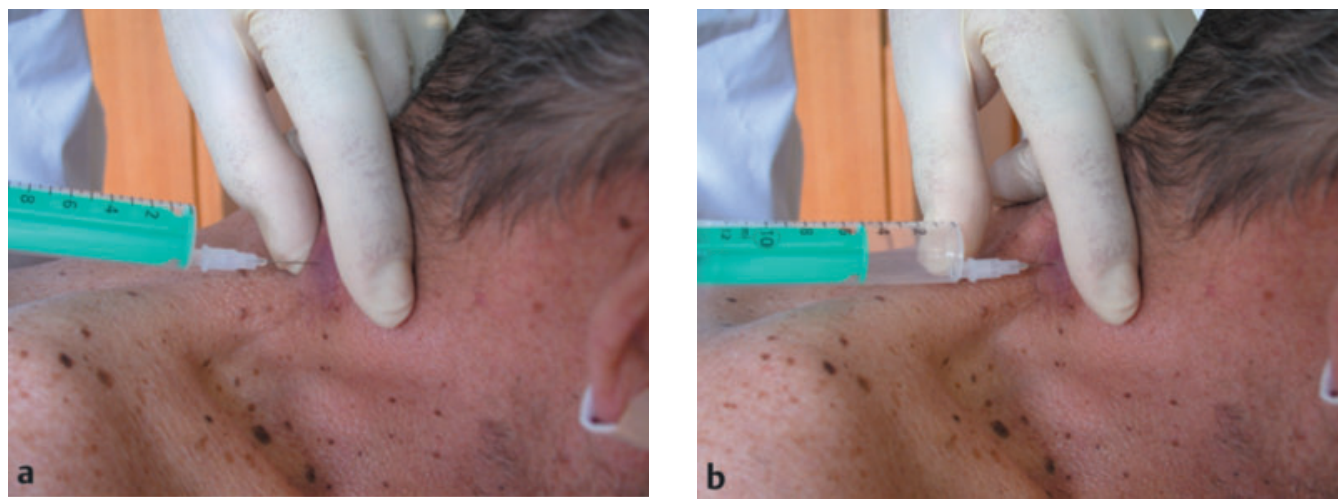

Abb. 2 Punktion palpabler Lymphknoten. (a) Fixieren des Lymphknotens und Einstechen in die Mitte der Läsion. (b) Anschließend fächerförmiges „Durchkämmen“ unter anhaltendem Sog.

Risiken und Vorbereitung I Die Punktion oberflächlich gelegener Lymphknoten verursacht kaum Schmerzen. Risiken für den Patienten ergeben sich praktisch nicht. Bei der Punktion nicht palpabler Lymphknoten kann es durch Punktion eines benachbarten Blutgefäßes zu einer Blutung kommen. Da der Nadeldurchmesser sehr gering ist, lässt sie sich in der Regel problemlos stillen. In diesen Fällen sollten vor der Punktion Gerinnungswerte bestimmt bzw. bei Patienten unter oraler Antikoagulation der Quick-Wert auf mindestens $40 \%$ angehoben werden.

Das Absetzen eines Thombozytenaggregationshemmers ist nicht erforderlich [2].

Zur sorgfältigen Vorbereitung gehört ferner die Rasur behaarter Körperpartien.

\section{Oberflächlich gelegene, palpable Lymphknoten}

Materialgewinnung | Zuerst wird die Hautoberfläche desinfiziert. Anschließend fixiert man den Lymphknoten mit einer Hand ( $\bullet$ Abb. 2a). Nach dem Einstechen in das Zentrum des Lymphknotens wird durch Ziehen am Spritzenkonus ein Sog erzeugt und der Lymphknoten unter Beibehaltung des Sogs fächerförmig „durchkämmt“ ( Abb. 2b). Das „Durchkämmen“ des Lymphknotens ( Abb. 3) erhöht die Wahrscheinlichkeit, vom Tumor befallene Regionen zu erfassen.

Zur Regulation des Sogs kann auch ein Binder-Ventil verwendet werden, das zwischen Nadel und Spritze eingesetzt wird.

\section{Unter Ultraschallsicht: Tiefer gelegene Lymphknoten}

Materialgewinnung I Sofern man nicht über einen speziellen Punktionsschallkopf verfügt, sollte man einen Handschuh über den Schallkopf ziehen, um sterile Bedingungen zu wahren. Anschließend wird die Hautoberfläche desinfiziert und der Schallkopf aufgesetzt. Die Nadel wird unter Ultraschall-Sicht in das Zentrum des Lymphknotens eingestochen ( $\triangleright$ Abb. 4 und $\triangleright$ Abb. 5 ). Nach Entfernen des Mandrins wird die Spritze aufgesteckt und durch Ziehen am Spritzenkonus ein Sog hergestellt. Anschließend aspiriert man fächerförmig lymphatisches Material.

Cave Vor dem Herausziehen der Nadel muss der Konus losgelassen werden, damit das aspirierte Material nicht in die Spritze gesaugt wird.

\section{Materialverarbeitung}

Ausstrich | Nach der Punktion wird das aspirierte Material ( $\bullet$ Abb. 6) auf einen Objektträger gespritzt und ausgestrichen $(\triangleright$ Abb. 7). Entsprechend den "Gepflogenheiten der Zytopathologen,

Abb. 3 Schema des fächerförmigen Durchkämmens des Lymphknotens.

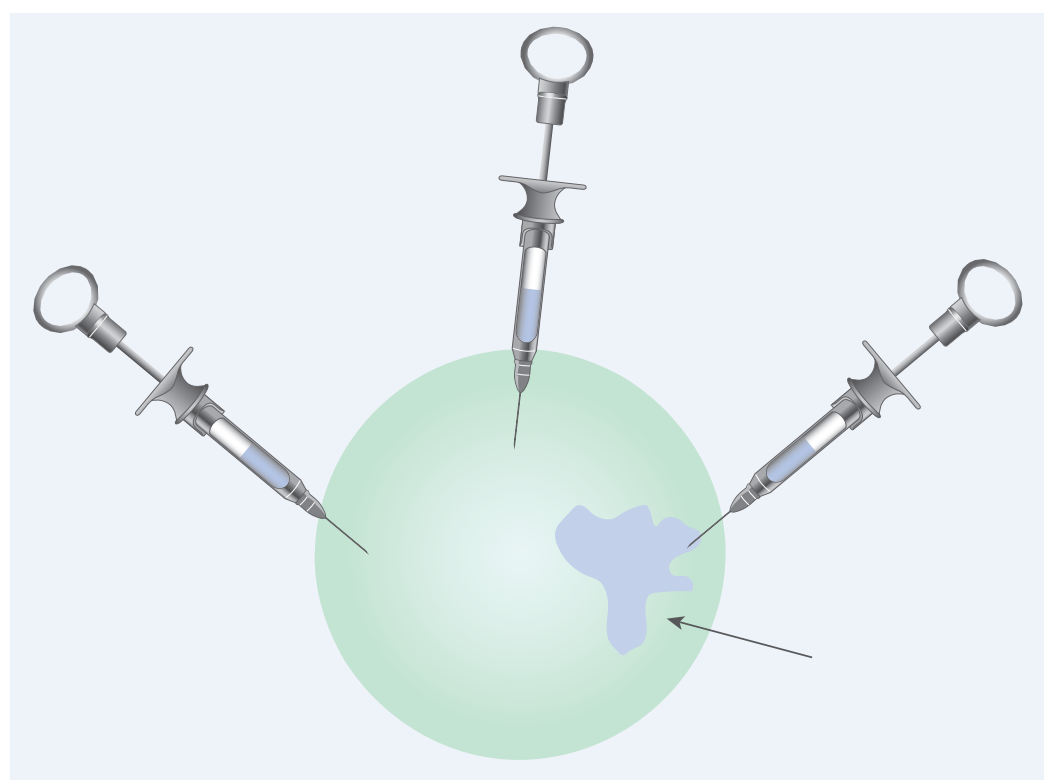




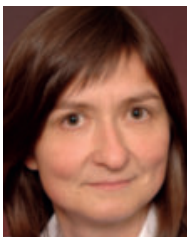

Dr. med. Sylvia Gütz ist Chefärztin der Kliniken für Pneumologie und Kardiologie am Ev. Diakonissenkrankenhaus Leipzig. sylvia.guetz@ediacon.de

Dipl.-Med. Thomas Knappe ist leitender Oberarzt der Klinik für Pneumologie am Ev. Diakonissenkrankenhaus Leipzig.

thomas.knappe@ediacon.de

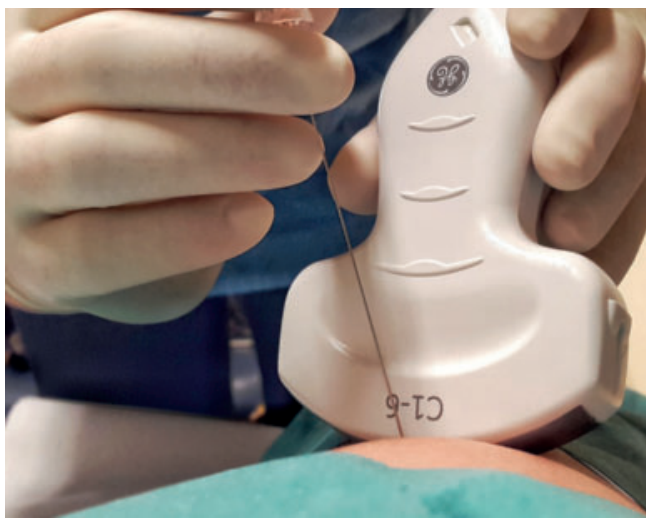

Abb. 4 Punktion in der Tiefe gelegener peripherer Lymphknoten: Einstellen der Läsion, Einstechen unter Ultraschall-Sicht.

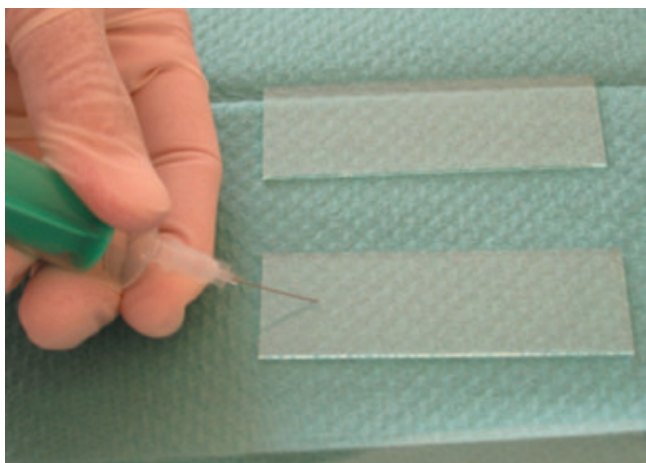

Abb. 6 Die Nadel wird auf einen Objektträger flach aufgelegt und der Nadelinhalt ausgespritzt.

mit denen man zusammenarbeitet, werden die Ausstriche luftgetrocknet bzw. fixiert gefärbt und mikroskopisch beurteilt.

\section{Konsequenz für Klinik und Praxis}

- Die Feinnadelpunktion peripherer Lymphknoten ist eine einfache Methode, die eine rasche Aussage zu Ätiologie und Dignität peripherer Lymphknotenschwellungen gestattet.

- Oberflächliche, palpable Lymphknoten lassen sich blind punktieren, tiefer gelegene mit größerer Sicherheit unter Ultraschallsicht.

- Verwendet werden Feinnadeln mit einer Stärke zwischen 20 und 26 Gauge.

- Nachdem die Nadel in den Lymphknoten eingestochen wurde, wird durch Ziehen am Spritzenkonus oder mit Hilfe eines Binder-Ventils ein Sog hergestellt und der Lymphknoten fächerförmig durchkämmt.

- Vor dem Herausziehen der Nadel ist der Sog zu beenden.

- Das aspirierte Material wird auf einem Objektträger ausgestrichen, luftgetrocknet bzw. fixiert und gefärbt. Nach einer Bearbeitungszeit von max. 20 min kann man es mikroskopisch beurteilen.

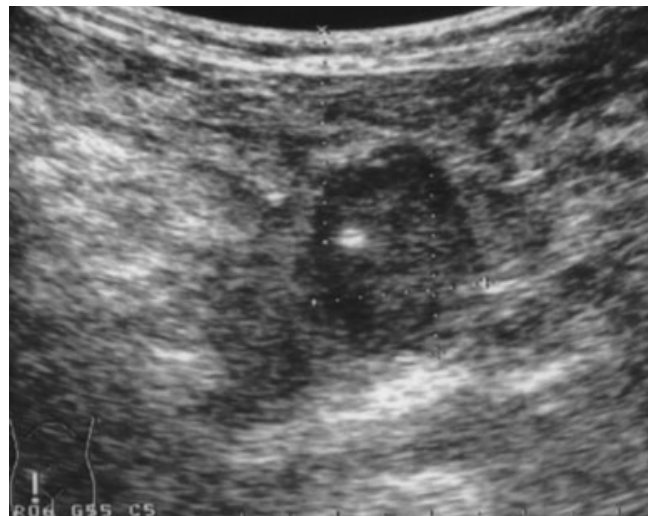

Abb. 5 Supraklavikulärer Lymphknoten (Mitte). Der weiße Punkt entsteht durch das „Nadelecho“ und zeigt, dass sich die Nadelspitze im Lymphknoten befindet.

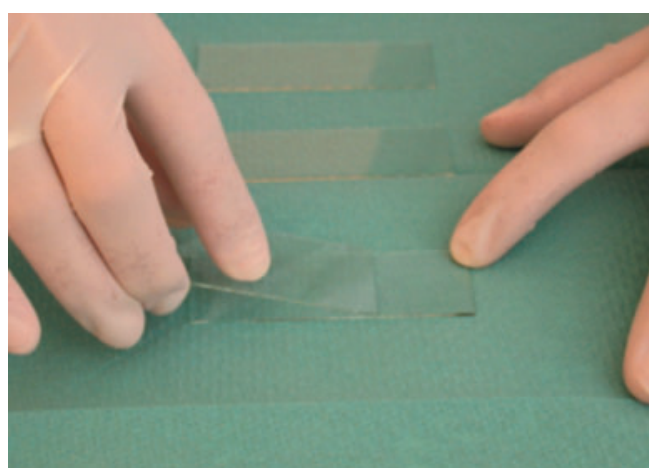

Abb. 7 Vorsichtiges Ausstreichen des Punktates mit Deckglas oder zweitem Objektträger.

\section{Literatur}

1 Chau I, Kelleher MT, Cunningham D et al. Rapid access multidisciplinary lymph node diagnostic clinic: analysis of 550 patients. $\mathrm{Br}$ J Cancer 2003; 88: 354-361

2 Carson H, Candel AG, Gattuso P, Castelli MJ. Fine-needle aspiration of supraclavicular lymph nodes. Diagn Cytopath 1996; 14: 216-220

3 Das DK. Value and limitations of fineneedle aspiration cytology in diagnosis and classification of lymphomas: A review. Diagn Cytopath 1999; 21: $240-249$

4 Stiegler R, Gosse H, Leonhardt P et al. Feinnadelaspirationszytologie als diagnostische bed-sideMethode zur Differenzialdiagostik peripherer Lymphknotenschwellungen. Pneumologie 2003; 57: 322-327

5 Wakely PE Jr. Fine-needle aspiration cytopathology in diagnosis and classification of malignant lymphoma: Accurate and reliable? Diagn Cythopath 2000; 22: 120-125w

\section{Interessenkonflikt}

Die Autoren geben an, dass kein Interessenkonflikt besteht. 